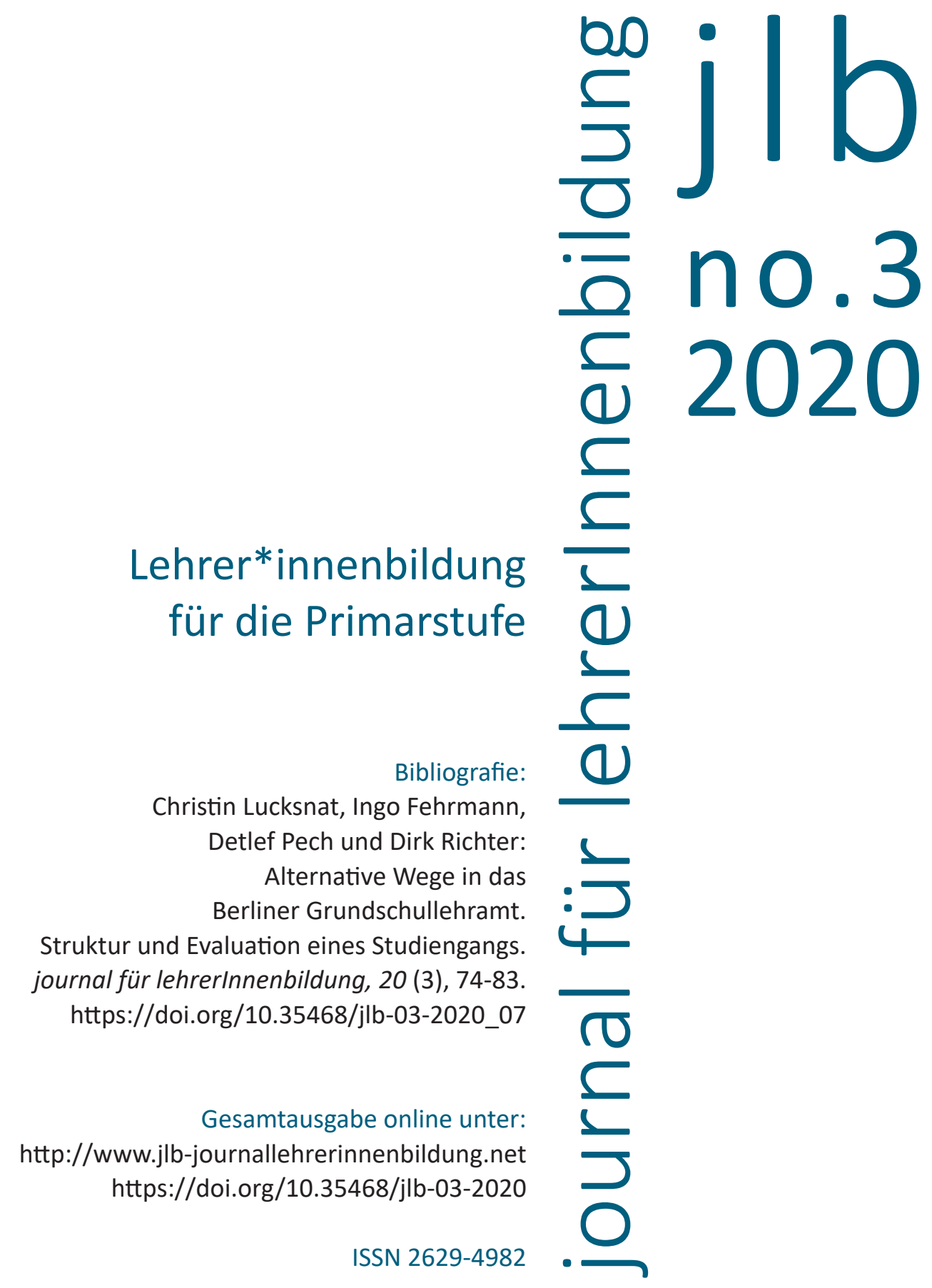


Christin Lucksnat, Ingo Fehrmann, Detlef Pech und Dirk Richter

\section{Alternative Wege in das Berliner Grundschullehramt. Struktur und Evaluation eines Studiengangs}


Aktuell stehen die Bildungsverwaltungen in Deutschland vor der Herausforderung, Stellen an Schulen nicht mehr hinreichend durch regulär qualifizierte Lehrkräfte besetzen zu können. Um diesem Lehrkräftemangel zu begegnen, werden verstärkt Personen ohne reguläres Lehramtsstudium eingesetzt (Quer- und Seiteneinsteiger*innen) (KMK, 2019). Berlin ist besonders stark vom Lehrkräftemangel betroffen: Während zu Beginn des Schuljahres 2018 laut KMK (2019) etwa 40 Prozent der neu eingestellten Lehrkräfte Quer- bzw. Seiteneinsteiger*innen waren, lag die Zahl zu Beginn des Schuljahres 2019 bereits bei 60 Prozent (Leinemann, 2019). Innerhalb Berlins stehen dabei die Grundschulen ${ }^{1}$ besonders im Fokus, da hier nicht genügend Lehrkräfte für den Vorbereitungsdienst ausgebildet wurden (Richter, Marx \& Zorn, 2018). Die Humboldt-Universität zu Berlin begegnet diesem Mangel an Berliner Grundschulen mit einer Neuerung, einem sogenannten Quereinstiegsmaster. Seit 2018 ermöglicht sie auch Personen mit einem Hochschulabschluss ohne Lehramtsbezug ein Vollzeit-Masterstudium „Lehramt an Grundschulen“ mit dem Abschlussziel Master of Education. Damit ist es den Absolvent*innen möglich, den regulären Vorbereitungsdienst zu durchlaufen und anschließend als regulär qualifizierte Lehrkräfte die Berufstätigkeit zu beginnen. Vergleichbare Modelle gibt es bisher außerhalb Berlins nicht (vgl. aber Caspari, 2019, für ein ähnliches Modell in Bezug auf das Lehramt an Sekundarschulen). Der Quereinstieg ins Lehramt ist dabei jedoch keine für Berlin spezifische Thematik. Auch in anderen deutschen Bundesländern sowie in der Schweiz und Österreich wird die Thematik derzeit stark diskutiert und beforscht (z. B. Abs, Anderson-Park \& Morgenroth, 2019; Riedl, 2019; Melzer, Pospiech \& Gehrmann, 2014). Es besteht somit ein hoher Bedarf zur Prüfung und Evaluation neu eingerichteter Qualifizierungsprogramme. Im vorliegenden Beitrag wird daher zunächst die Struktur der grundschulbezogenen Lehramtsstudiengänge an der Humboldt-Universität zu Berlin sowie im Anschluss das laufende Evaluationsprojekt vorgestellt.

1 In Berlin und Brandenburg ist die Grundschule auf sechs Jahre angelegt. Dies unterscheidet sich von allen anderen Bundesländern, in denen die Grundschule nur vier Jahre umfasst. 


\section{Studienstruktur für das Lehramt an Grundschulen}

Im Jahr 2014 wurde im Land Berlin die Lehrkräftebildung komplett neu geregelt. Seitdem stehen erstens ausdrücklich berufsbezogene Kompetenzen im Fokus, zweitens wird der Lehrberuf ausdrücklich als Profession verstanden (vgl. LBiG, 2014, § 1). Des Weiteren zeichnet sich der Studiengang dadurch aus, dass statt eines Studiums der Grundschulpädagogik mit integrierten Lernbereichen ein Fachstudium in drei Fächern absolviert wird (LZVO, 2014, Anlage 1). Entsprechend der KMK-Rahmenvereinbarung für den Lehramtstyp 1 (KMK, 1997) gehören Deutsch und Mathematik zu den zu belegenden Pflichtfächern. Nach einem insgesamt fünfjährigen Studium wird der Abschluss Master of Education erworben. Im Ergebnis führt dies zu einer deutlichen Stärkung der fachdidaktischen und fachwissenschaftlichen Studienanteile.

Die Studierenden des Quereinstiegsmasters (Q-Master) erwerben ihre Lehrbefähigung in den Fächern Deutsch, Mathematik und Sachunterricht. Andere Fächerkombinationen werden im Q-Master nicht angeboten. Der Aufbau des Q-Master-Studiums unterscheidet sich dabei nicht vom regulären Masterstudium für das Lehramt an Grundschulen. In beiden Studiengängen liegt der Schwerpunkt auf bildungswissenschaftlichen Inhalten und dem Praxissemester. Mit mindestens 18 Leistungspunkten pro Fach ist der Anteil an fachlichen und fachdidaktischen Inhalten deutlich geringer als im lehramtsbezogenen Bachelorstudium (42 Leistungspunkte pro Fach). Aufgrund dieses Unterschieds müssen die Studierenden des Q-Masters schon vor Beginn ihres Studiums fachwissenschaftliche und fachdidaktische Leistungen in allen drei Studienfächern nachholen, sofern diese noch nicht erbracht wurden. Dieser Nachweis erfolgt über ein zweisemestriges Zertifikatsstudium („Zertifikatsstudium Deutsch - Mathematik - Sachunterricht an Grundschulen“, intern "Q-Plus" genannt), in dem je nach Erststudium in einem, zwei oder allen drei Fächern noch die nötigen Studienleistungen für die Bewerbung zum Q-Master-Studium nachgeholt werden.

Es gibt somit an der Humboldt-Universität zwei Wege zur Erreichung eines Master-of-Education-Abschlusses für das Lehramt an Grundschulen: Entweder über das Bachelorstudium „Bildung an Grundschulen“ mit anschließendem regulärem Masterstudium oder, bei Vorlie- 
gen eines ersten Hochschulabschlusses, über das Zertifikatsstudium (Q-Plus) und das Q-Master-Studium. Bisher ist allerdings wenig über die Wirksamkeit von universitären Qualifizierungsmaßnahmen für nicht-traditionell (also ohne Abschluss eines lehramtsbezogenen Bachelorstudiums) qualifizierte Lehrkräfte bekannt. Aus diesem Grund werden derzeit die beiden Masterstudiengänge für das Grundschullehramt an der HU Berlin vergleichend in einem Forschungsprojekt evaluiert.

\section{Evaluation der Studiengänge an der Humboldt-Universität}

Mit Beginn des Wintersemesters 2019/20 werden die beiden Masterstudiengänge für das Lehramt an Grundschulen - also das reguläre Masterstudium und der Q-Master - in einer längsschnittlich angelegten Untersuchung über zwei Jahre in einem Kooperationsprojekt der Universität Potsdam, der Bertelsmann Stiftung und der HU Berlin evaluiert, wobei der Fokus dabei auf dem Fach Sachunterricht und seiner Didaktik liegt. Der Fokus wurde insbesondere auf dieses Fach gelegt, da es bisher kaum Forschung zur Professionalisierung von Lehrkräften im Fach Sachunterricht gibt und damit eine Lücke geschlossen werden soll. Durch die Evaluation sollen zwei übergeordnete Fragen beantwortet werden: Zum einen soll untersucht werden, ob sich reguläre Studierende und Q-Master-Studierende hinsichtlich a) ihrer Ausgangsbedingungen und b) ihrer professionellen Kompetenz unterscheiden. Zum anderen soll der Frage nachgegangen werden, wie sich die professionelle Kompetenz der regulären Studierenden und der QMaster-Studierenden im Verlauf des Studiums entwickelt. Insgesamt nimmt die Evaluation zwei verschiedene Kohorten in den Blick. Die erste Kohorte umfasst Q-Master-Studierende sowie reguläre Lehramtsstudierende, die ihr Masterstudium mit Beginn des Wintersemesters 2019/20 aufgenommen haben. Daneben wird eine weitere Kohorte von Studierenden des Q-Masters betrachtet, die den Studiengang im Wintersemester 2020/21 beginnt, aber bereits im Oktober 2019 das Zertifikatsstudium begonnen hat (Q-Plus-Studierende).

In Anlehnung an das Kompetenzmodell von Baumert und Kunter (2006) werden durch Tests und Befragungen das Professionswissen, 
Überzeugungen, motivationale Orientierungen und selbstregulative Fähigkeiten der Studierenden erfasst. Dabei werden sowohl allgemeine als auch fachspezifische Kompetenzen exemplarisch für das Fach Sachunterricht untersucht. Zur Beschreibung der Kompetenzentwicklung der verschiedenen Studierendengruppen werden Tests und Befragungen zu drei verschiedenen Messzeitpunkten eingesetzt: zu Beginn ihres Studiums, zur Mitte nach einem Jahr sowie zum Ende des Studiums.

Über den quantitativen Forschungsansatz hinaus werden auch Interviews mit einer kleinen Auswahl von ca. 15 Studierenden geführt, die zu gleichen Anteilen jeweils aus einer der beiden Q-Master-Kohorten und dem regulären Studiengang ausgewählt werden. Ziel der Interviews ist es, genauere Erkenntnisse über die Erwartungen an und die Erfahrungen mit den jeweiligen Studiengängen zu erhalten. Die Interviews sollen somit Informationen liefern, die zur Kontextualisierung der Befunde aus den Befragungen und Tests beitragen.

\section{Erster Vergleich der Studierendengruppen}

Zum ersten Messzeitpunkt haben bisher 111 Studierende an den Tests und Befragungen teilgenommen, von denen 54 Personen den regulären Master, 24 Personen den Q-Master und 23 Personen das Zertifikatsstudium (Q-Plus) absolvieren. Ein detaillierter Blick auf die Gruppe der Personen im regulären Master zeigt, dass eine Vielzahl der Lehramtsstudierenden bereits vor dem lehramtsbezogenen Bachelorstudium eine Ausbildung oder ein anderes Hochschulstudium abgeschlossen hat. Diese Gruppe, die hier als Lehramtsstudierende mit Vorberuf bezeichnet wird, macht in dieser Stichprobe mehr als die Hälfte der Studierenden im regulären Master aus.

Ein differenzierter Blick auf die Gruppen zeigt, dass Lehramtsstudierende mit Vorberuf, Q-Master-Studierende und Q-Plus-Studierende mit durchschnittlich 34 Jahren knapp 10 Jahre älter sind als die regulären Lehramtsstudierenden ohne Vorberuf, die vorab keine andere berufliche Qualifizierung durchlaufen haben. In der Geschlechterverteilung zeigten sich keine Unterschiede zwischen den Gruppen. Hinsichtlich des vorherigen Bildungsweges zeigte sich, dass Lehramtsstudierende mit Vorberuf vor ihrem lehramtsbezogenen Bachelorstudium häufiger eine Ausbildung abgeschlossen haben, während Q-Master- und 
Q-Plus-Studierende über verschiedene Arten von Studienabschlüssen verfügen (siehe Abb. 1). Etwa 20 Prozent der Lehramtsstudierenden mit Vorberuf haben eine Ausbildung als Erzieher*in absolviert. Darüber hinaus verfügen etwa 31 Prozent der Lehramtsstudierenden mit Vorberuf bereits vor ihrem lehramtsbezogenen Bachelorstudium über einen ersten Studienabschluss, d. h. viele Personen mit erstem Hochschulabschluss haben auch schon vor Einführung des Q-Masters ein grundschulbezogenes Lehramtsstudium aufgenommen.

Die Mehrheit der Q-Master- und Q-Plus-Studierenden (96 Prozent) berichten über pädagogische Vorerfahrungen vor dem Q-Master bzw. Zertifikatstudium, vor allem durch Schülernachhilfe (79 Prozent), durch eine sonstige berufliche Tätigkeit in der Kinder- und Jugendarbeit (52 Prozent), durch Kinder- und Jugendarbeit im Freizeitbereich (47 Prozent) oder durch eine Tätigkeit als Vertretungslehrkraft (21 Prozent) $)^{2}$.

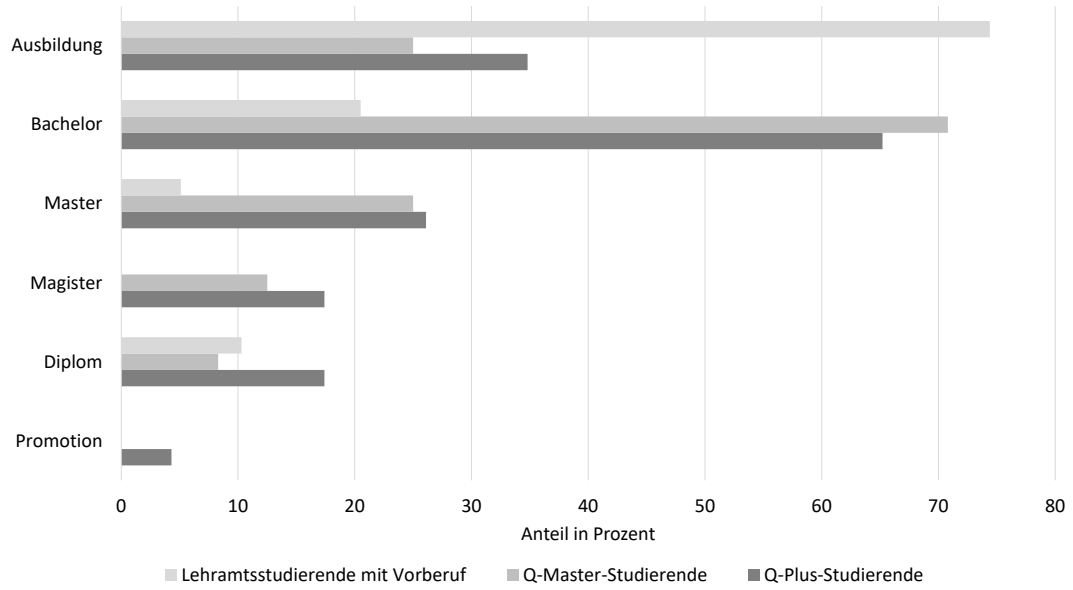

Abb. 1 Anteile der Personen in der Stichprobe, die vor Beginn des Studiums eine Ausbildung oder ein anderes Studium absolvierten (Anmerkungen: Lehramtsstudierende mit Vorberuf $\mathrm{N}=39$, Q-Master-Studierende $\mathrm{N}=24$, Q-PlusStudierende $\mathrm{N}=23$ )

2 Da Personen mehrere pädagogische Vorerfahrungen berichten konnten, liegt die Summe der Einzelangaben bei über 100 Prozent. 


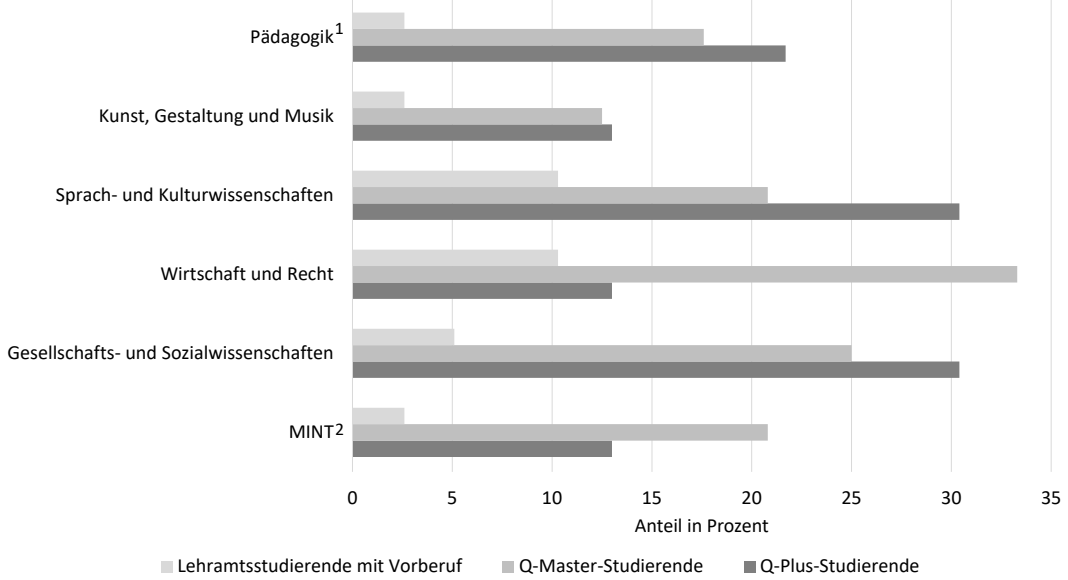

Abb. 2 Anteile der Personen, die vor Beginn des Studiums ein anderes Fachstudium absolvierten (Anmerkungen: Lehramtsstudierende mit Vorberuf $\mathrm{N}=39$, Q-Master-Studierende N=24, Q-Plus-Studierende $\mathrm{N}=23$; ${ }^{1}$ Zu dieser Kategorien gehören Studienfächer wie z. B. soziale Arbeit oder Erziehungswissenschaften; ${ }^{2}$ Studienfächer der Mathematik, Informatik, Naturwissenschaften und Technik)

\section{Fazit}

Der Quereinstiegsmaster an der HU Berlin eröffnet einen neuen Zugang ins Lehramt für Personengruppen, die andere Hintergründe aufweisen und andere Erfahrungen mitbringen als traditionell ausgebildete Lehramtsstudierende. Die ersten Ergebnisse der Evaluation des Studiengangs haben gezeigt, dass Student*innen des Q-Masters bereits älter sind und über mindestens einen nicht-lehramtsbezogenen Hochschulabschluss verfügen. Zudem zeigt sich der positive Befund, dass die Studierenden des Q-Masters und des Zertifikatstudiums bereits vor Aufnahme des Q-Masters über wichtige pädagogische Vorerfahrungen verfügen und auf diese aufbauen können. Darüber hinaus gibt es auch unter den regulären Lehramtsstudierenden solche, die bereits vor ihrem Studium einen Beruf erlernt haben. Es stellt sich die Frage, inwiefern sich diese Gruppe von Quereinsteiger*innen unterscheidet. Es ist anzunehmen, dass reguläre Lehramtsstudierende mit Vorberuf über mehr pädagogisches Wissen verfügen als die Quereinsteiger*innen, da diese keine pädagogischen Seminare im 
Zertifikatstudium belegen. Reguläre Lehramtsstudierende mit Vorberuf haben diese hingegen im Rahmen ihres lehramtsbezogenen Bachelorstudiums absolviert. Es wäre allerdings zu vermuten, dass beide Gruppen aufgrund ihrer vorherigen beruflichen Erfahrungen wichtige Fähigkeiten zum Umgang mit Herausforderungen und möglichen Stressfaktoren des Lehrberufs mitbringen (Troesch \& Bauer, 2017).

Die Evaluation des Q-Masters weist hinsichtlich des Studiendesigns Ähnlichkeiten zu bereits abgeschlossenen Evaluationen von Quereinsteigerprogrammen, wie z. B. dem QUER-Programm an der TU Dresden auf. So erfassen auch wir u.a. das pädagogische Wissen, pädagogische Vorerfahrungen und die Berufswahlmotivation der verschiedenen Gruppen. Darüber hinaus untersucht die Evaluation des Q-Masters an der HU Berlin im Gegensatz zum QUER-Programm an der TU Dresden auch andere Kompetenzfacetten- und -aspekte, wie z. B. selbstregulative Fähigkeiten und erfasst darüber hinaus das fachliche und fachdidaktische Wissen nicht durch Selbstberichte, sondern durch Tests.

Abschließend lässt sich feststellen, dass wir bisher noch zu wenig über die Gruppe der Quereinsteiger*innen wissen. Es stellen sich wichtige Fragen hinsichtlich der Kompetenzen, die Quereinsteiger*innen mitbringen und wie sich diese im Verlauf entwickeln. Die Evaluation des Studiengangs hat zum Ziel, einen Beitrag zur Beantwortung dieser Fragen zu leisten. Testungen und Befragungen zu mehreren Zeitpunkten im Masterstudium sollen Antworten darauf geben, mit welchen Kompetenzen die Q-Master-Studierenden beginnen und wie sich diese bis zum Abschluss des Studiengangs entwickeln.

\section{Literatur}

Abs, H. J., Anderson-Park, E. \& Morgenroth, S. (2019). Recruiting and Preparing Teachers Through an Alternative Programme: A European Policy Experiment on the Teach For All Approach in Five Countries. University of Duisburg-Essen.

Baumert, J. \& Kunter, M. (2006). Stichwort: Professionelle Kompetenz von Lehrkräften. Zeitschrift für Erziehungswissenschaft, 9 (4), 469-520.

Caspari, D. (2019). Der Q-Master an der Freien Universität Berlin: ein Konzept für einen individuelle(re)n Weg in den Lehrberuf. journal für lehrerInnenbildung, 19 (2), 66-72.

KMK [Kultusministerkonferenz] (1997). Rahmenvereinbarung über die Ausbildung und Prüfung für ein Lehramt der Grundschule bzw. Primarstufe (Lehramtstyp 1). Beschluss der Kultusministerkonferenz vom 28.02.1997 i. d. F. vom 14.03.2019. 
Verfügbar unter https://www.kmk.org/fileadmin/Dateien/veroeffentlichungen_ beschluesse/1997/1997_02_28-RV_Lehramtstyp_1.pdf [23.12.2019].

KMK (2019). Einstellung von Lehrkräften 2018. Berlin: KMK.

LBiG [Lehrkräftebildungsgesetz] (2014). Gesetz über die Aus-, Fort- und Weiterbildung der Lehrerinnen und Lehrer im Land Berlin (Lehrkräftebildungsgesetz - LBiG) vom 7. Februar 2014. In GVBI. 2014, 49. Verfügbar unter http://gesetze.berlin.de/jpor tal/?quelle=jlink\&query=LehrBiG+BE\&psml=bsbeprod.psml\&max=true\&aiz=true [23.12.2019].

Leinemann, S. (01.08.2019). Lehrermangel: Berlin setzt weiterhin auf Quereinsteiger. Berliner Morgenpost. Verfügbar unter: https://www.morgenpost.de/berlin/ article226652987/Lehrermangel-Berlin-setzt-weiterhin-auf-Quereinsteiger.html [13.07.2020].

LZVO [Lehramtszugangsverordnung] (2014). Verordnung über den Zugang zu Lehrämtern (Lehramtszugangsverordnung - LZVO) vom 30. Juni 2014. In GVBI. 2014, 242. Verfügbar unter http://gesetze.berlin.de/jportal/?quelle=jlink\&query=LehrZV+BE \&psml=bsbeprod.psml\&max=true\&aiz=true [23.12.2019].

Melzer, W., Pospiech, G. \& Gehrmann, A. (2014). QUER - Qualifikationsprogramm für Akademiker zum Einstieg in den Lehrerberuf. Abschlussbericht Dezember 2014. Dresden.

Richter, D., Marx, A. \& Zorn, D. (2018). Lehrkräfte im Quereinstieg: sozial ungleich verteilt? Eine Analyse zum Lehrermangel an Berliner Grundschulen. Gütersloh: Bertelsmann Stiftung.

Riedl, A. (2019). Theorie-Praxis-Verzahnung in einem Masterstudium mit Referendariat. Professionalisierung von Quereinsteiger*innen. journal für lehrerlnnenbildung, 19 (2), 58-65.

Troesch, L. M. \& Bauer, C. E. (2017). Second career teachers: Job satisfaction, job stress, and the role of self-efficacy. Teaching and Teacher Education, 67, 389-398. 
Christin Lucksnat, Mitarbeiterin am Lehrstuhl für Erziehungswissenschaftliche

Bildungsforschung, Universität Potsdam.

Arbeitsschwerpunkte:

Kompetenzen und Kompetenzentwicklung von Quer- und Seiteneinsteiger*innen

lucksnat@uni-potsdam.de

Ingo Fehrmann, Dr., wiss. Mitarbeiter an der Humboldt-Universität zu Berlin, Koordinator des Quereinstiegsmasterstudiums

Lehramt an Grundschulen.

Arbeitsschwerpunkte:

Sprachdidaktik, Unterrichtsbeobachtung

ingo.fehrmann@hu-berlin.de
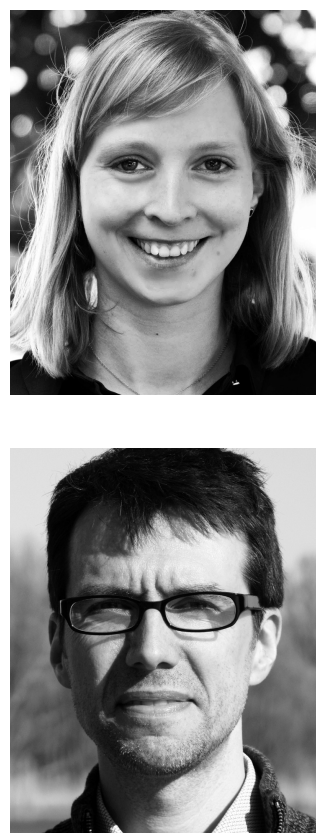

Detlef Pech, Dr., Professor am Institut für Erziehungswissenschaften, Humboldt-Universität zu Berlin.

Arbeitsschwerpunkte: Inklusion und Fachdidaktik, Sachunterrichtsdidaktik und Bildung, Kindheitsforschung

detlef.pech@hu-berlin.de

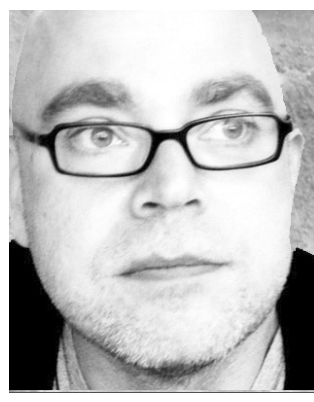

Dirk Richter, Dr., Professor am Department für Erziehungswissenschaft, Universität Potsdam. Arbeitsschwerpunkte: Kompetenzentwicklung von Lehrkräften in der Aus- und Fortbildung, Nutzung digitaler Medien beim beruflichen Lernen, Evaluationen beruflicher Lerngelegenheiten

dirk.richter@uni-potsdam.de

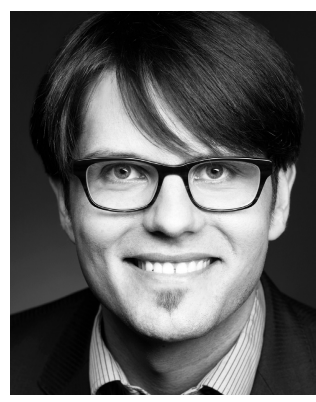

